\title{
A describing function approach to bipolar RF-power amplifier simulation
}

\author{
Vidkjær, Jens
}

Published in:

IEEE Transactions on Circuits and Systems

Link to article, DOI:

10.1109/TCS.1981.1085047

Publication date:

1981

Document Version

Publisher's PDF, also known as Version of record

Link back to DTU Orbit

Citation (APA):

Vidkjær, J. (1981). A describing function approach to bipolar RF-power amplifier simulation. IEEE Transactions on Circuits and Systems, 28(8), 758-767. https://doi.org/10.1109/TCS.1981.1085047

\section{General rights}

Copyright and moral rights for the publications made accessible in the public portal are retained by the authors and/or other copyright owners and it is a condition of accessing publications that users recognise and abide by the legal requirements associated with these rights.

- Users may download and print one copy of any publication from the public portal for the purpose of private study or research.

- You may not further distribute the material or use it for any profit-making activity or commercial gain

- You may freely distribute the URL identifying the publication in the public portal

If you believe that this document breaches copyright please contact us providing details, and we will remove access to the work immediately and investigate your claim. 


\title{
A Describing Function Approach to Bipolar RF-Power Amplifier Simulation
}

\author{
JENS VIDKJAER, MEMBER, IEEE
}

\begin{abstract}
A method for fast and accurate computations of the primary performance parameters such as gain, efficiency, output power, and bandwidth in class- $C$ biased RF-power amplifier stages is presented. The method is based on a describing function characterization of the RF-power transistor where the terminal currents are assumed sinusoidal. The approximation comprises both the input and output properties of the transistor simultaneously and includes the effects of base widening, current saturation, and the most significant thermal dependencies. The method is verified through a series of experiments.
\end{abstract}

\section{INIRODUCTION}

$\mathrm{T}$ HE LACK OF analytical and computerized aids in the field of RF-power amplifier design is widely recognized. Time-domain simulations are possible, but the computational costs may be prohibitive for their use as common engineering tools. The reason is that the underlying transistor models must be rather complex if the simulated results are to show that high degree of accuracy which, unfortunately, is required in RF design, cf., [1]. Moreover, the needs are often in the form of results over a parameterdomain, for instance frequency, loading, temperature, etc., so repeated use of the time-domain approach is required before the necessary information is gathered.

This paper presents a simulation method which more directly suits the requirements for a design tool. Within its range of coverage it provides fast and accurate results in the terms needed for engineering decisions. The method is based on a describing function characterization of the transistor and benefits from the fact, that within this framework it is possible accurately to take into account the thermal effects caused by the self-heating of the transistor. This is of great importance in high efficiency, high power circuits. On the other hand, the approach also implies some fundamental assumptions:

A) The amplifier has a structure, as shown in Fig. 1.

B) The ac portions of the transistor terminal currents are of sinusoidal shapes.

In addition a scries of simplifications concerning the transistor modeling must be introduced if the computations shall possess significant improvements in speed. These simplifications are made from the point of view that:

C) The power transistor is intended to operate near its rated performance with respect to power, efficiency, and frequency.

Manuscript received September 25, 1980; revised January 31, 1981 , This work was supported by a Grant from the Danish Council for Scientific and Industrial Research.

The author is with the Electronics Institute, Technical University of Denmark, DK-2800 Lyngby, Denmark.

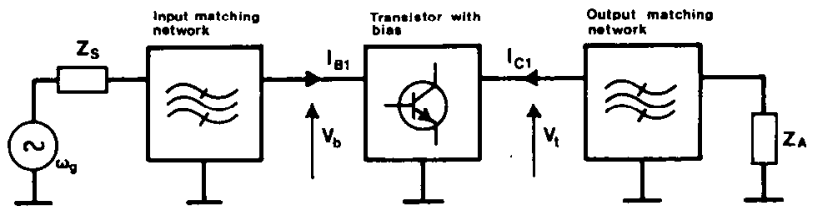

Fig. 1. Structure of the RF-power amplifier to be considered. The interfacing currents $I_{B 1}$ and $I_{C 1}$ are assumed sinusoidal.

By conditions A) to C) the type of amplifiers to be considered covers the majority of output stages in VHF and UHF transmitters for mobile communications. In agreement with $\mathrm{A}$ ) and $\mathrm{B}$ ), the series method of tuning the transistor has settled in practice in such circuits. This may be understood from the observation that in order to obtain condition C), the transistor should be driven so hard that a significant base widening accompanies its current conduction. This point will be further considered below, but it is in full agreement with a detailed time-domain investigation of the amplifiers which has been previously reported [2]. As the base widening occurs under operational conditions which conventionally are ascribed to saturation, the effect is strongly facilitated by the series tuning method which has evolved through experience.

\section{Transistor Modeling Fundamentals}

In the case where base widening controls the current conduction of the transistor its two major consequences are [2]:

1) Base widening prevents the transistor from becoming saturated in the traditional sense.

2) Base widening modulates the collector series resistance.

The mechanism behind 1) is that the amount of charge, which is injected through the base terminal in excess of the requirements for turning the transistor on, is absorbed in the base-emitter capacitance. This is in contrast to the common perception of saturation where such a charge is stored in the base-collector capacitance. As base widening furthermore implies the collapse of the collector built-in field [3], the junction voltage is fixed to the built-in potential $\Phi_{C}$ and the collector charge becomes accordingly clamped to a small amount $Q_{C 0}$. Therefore, no significant charging current flows through the base-collector capacitance so the whole collector current is led to the emitter terminal in the base widening controlled period. As, during the same period, the emitter junction is forward biased and approximately fixed to the level $V_{\mathrm{BE} 0}$, the current conduc- 


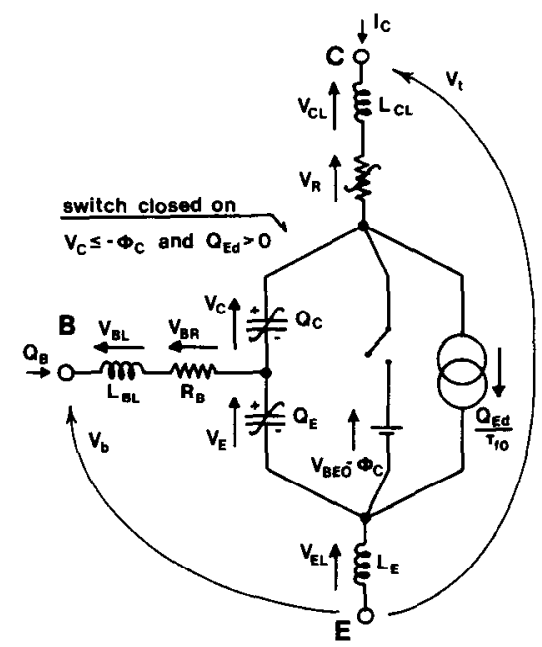

Fig. 2. Transistor equivalent diagram. The closing of the switch corresponds to base widening mode of operation. $Q_{\mathrm{Ed}}$ denotes the emitter diffusion charge which is contained in $Q_{E} . L_{C L}, L_{B L}, L_{E}$ denote the bonding and lead inductances and $R_{B}$ is the linearized base resistance. The nonlinear components are given by (1)-(4), (13)-(16), and (21).

tion may in this case be modeled by nothing more than an EMF of $V_{\mathrm{BE} 0}-\Phi_{C}$. This can be included in the transistor equivalent circuit by a controlled switch as shown in Fig. 2. Thereby a highly nonlinear phenomenon, which becomes rather complex in conventional models where a controlled current source is the only conducting element [2], [4], has been simplified much by structural changes in the equivalent circuit.

Considering the modulation of the collector series resistance, base widening acts in conjunction with the effect of current saturation. This may be expressed by [2]

$$
V_{R}=(1-\eta) V_{\mathrm{RS}}\left(I_{C}\right)
$$

where $V_{\mathrm{RS}}$ gives the saturation characteristic of the collector region and $\eta$ denotes the base widening ratio, i.e., the width of the base extension over the metallurgical collector width. An expression for $V_{\mathrm{RS}}$ cannot be derived from basic physical principles but several empirical relations are in use, for instance,

$$
V_{\mathrm{RS}}=\operatorname{sign}\left(I_{C}\right) R_{C 0} I_{\lim } \log \left[\frac{I_{\lim }}{I_{\lim }-\left|I_{C}\right|}\right], \quad\left|I_{C}\right|<I_{\text {lim }} .
$$

This expression shows the required asymptotic properties of ohmic conduction $I_{C} R_{C O}$ at low currents and a raise towards infinity as the current approaches the scattering limited boundary $I_{\mathrm{lim}}$. If base widening controls the current flow as discussed above, $\eta$ enters the relationship between the collector current and the emitter diffusion charge $Q_{\mathrm{Ed}}$ through [2],

$$
I_{C}=\frac{Q_{\mathrm{Ed}}}{\tau_{f 0}(1+Z \eta)^{2}}
$$

where $\tau_{f 0}$ is the forward transit time prior to base widening and $Z$ is a geometry and doping dependent quantity which is commonly difficult to estimate. However, it may be substituted by the more convenient maximum transit time

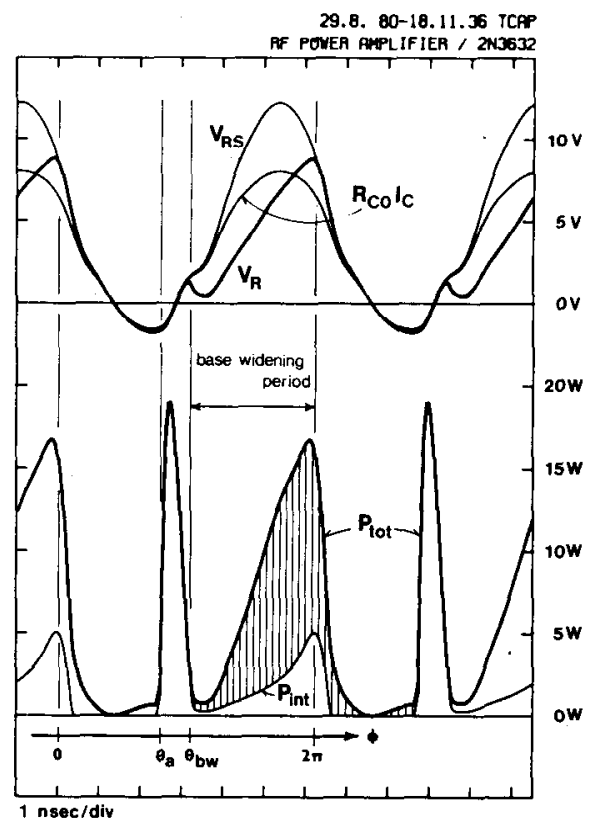

Fig. 3. Time-domain simulation of a RF-power amplifier (Fig. 8). The upper curves show the differences between the linear conduction $R_{C 0} I_{C}$, the saturation characteristic $V_{\mathrm{RS}}\left(I_{C}\right)$, and the final series resistance voltage $V_{R}$. The lower curves show the instant total transistor power dissipation $P_{\text {tot }}$ and the intrinsic dissipation $P_{\text {int }}$.

$\tau_{\max }$ and thereby the base widening ratio to be used in (1) becomes

$$
\eta=\sqrt{\frac{\mathcal{Q}_{\mathrm{Ed}}}{\tau_{x} I_{C}}}-\sqrt{\frac{\tau_{f 0}}{\tau_{x}}}, \quad 0 \leqslant \eta \leqslant 1
$$

with

$$
\tau_{x}=\tau_{\max }+\tau_{f 0}-2 \sqrt{\tau_{f 0} \tau_{\max }}, \quad \tau_{\max }=(1+Z)^{2} \tau_{f 0} .
$$

As $\eta$ for physical reasons is restricted to the interval $[0,1],(4)$ should be understood in the way that $\eta$ is clamped to the respective boundary if the expression provides a result outside the interval.

The impact of base widening upon RF-power amplifier performance is not widely recognized. As an illustration, therefore, Fig. 3 shows how the series voltage in (1) appears in a time-domain simulation. It is seen that the $V_{\mathrm{RS}}$ characteristic provides a significant excessive loss compared with the ohmic conduction. The net result in $V_{R}$, however, shows that base widening counteracts this. The collector series loss in VHF and UHF amplifiers may often be more than half the total transistor dissipation. This is demonstrated by the lower curves where the hatched area represents the total series loss in the transistor to which the collector contribution is completely dominating. Therefore, the balance between the two nonlinear effects is the most important consideration for determining the amplifier efficiency.

It should be noted that in full details the effect of base widening is much more complicated than the above presentation suggests, cf., [3]-[5]. However, (1) and (3) remain accurate if none of the boundaries $\eta \rightarrow 1$ or $\eta \rightarrow 0$ are approached. Since this is the dominating situation in the amplifiers under consideration, no attempts have been 


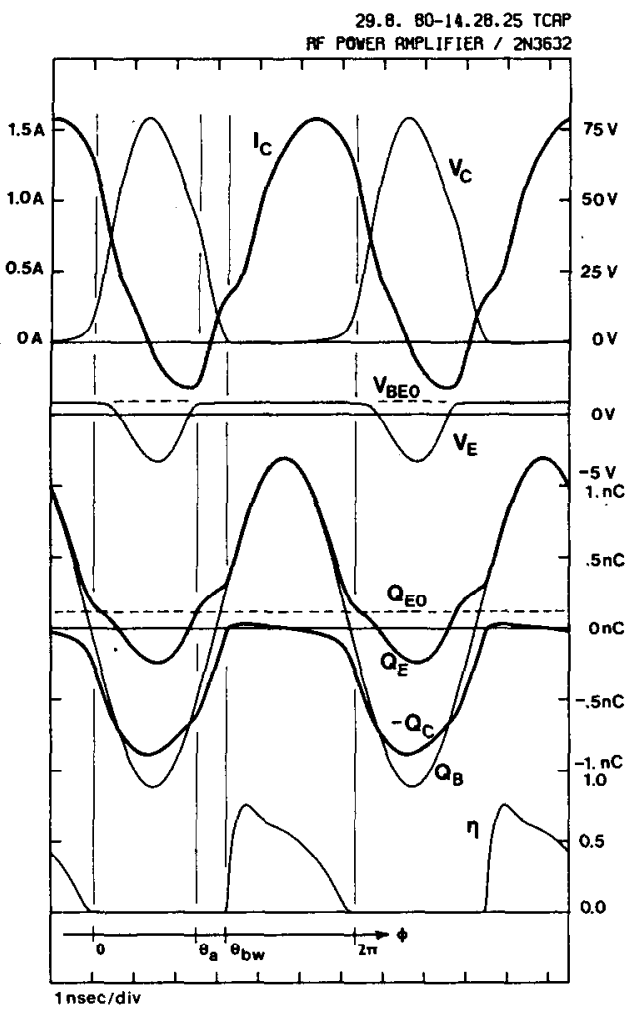

Fig. 4. Time-domain simulation of the amplifier in Fig. 8, showing the wave shapes of the variables which are most important for deriving the describing functions of the transistor.

made in order to go further with the modeling of the base widening.

Besides the abovementioned properties several other aspects regarding the formulation and utilization of the transistor equivalent circuit in Fig. 2 need consideration. Most of these will be taken up as the matters evolve. A major assumption should be mentioned initially: Only three consecutive transistor modes occur during an operation cycle of the amplifier. Using the phase variable $\varphi=\omega_{g} t$ these are

$$
\begin{array}{ll}
0 \leqslant \varphi \leqslant \theta_{a}: & \text { Cutoff } \\
\theta_{a} \leqslant \varphi \leqslant \theta_{\mathrm{bw}}: & \text { Forward active } \\
\theta_{\mathrm{bw}} \leqslant \varphi \leqslant 2 \pi: & \text { Base widening controlled current } \\
& \text { conduction. }
\end{array}
$$

Fig. 4 shows an example of how this subdivision applies to the wave-shapes of the most important transistor variables. The simplification is that the transistor is assumed to go directly from the base widening mode to the cutoff state without an intervening active period. This is seen to be a reasonable assumption in the example of Fig. 4. It is generally valid if the transit time is small compared with the period time, i.e.,

$$
\tau_{f 0} \omega_{g}<1
$$

and, simultaneously, the transistor is driven so hard that a significant base widening results. The validity of the assumption may, conversely, be used as an indication of whether or not the circuit is operated in accordance with assumption $\mathrm{C}$ ) above.

\section{DESCRIBING FunCtIONS FOR ThE TRANSISTOR Terminal Voltages}

The task to be undertaken in this section is to establish expressions or computing schemes whereby the mean-values and the first harmonic (fundamental frequency) components of the transistor terminal voltages may be calculated. The basis for this is the assumed sinusoidal terminal current wave shapes. The collector current is, therefore, expressed

$$
I_{C}(\varphi)=I_{C 0}+I_{C 1} \cos \left(\varphi-\theta_{C}\right) .
$$

At the base terminal it becomes more convenient to use the total base charge

$$
Q_{B}(\varphi)=Q_{E}(\varphi)-Q_{C}(\varphi)
$$

as the forcing function since charge control concepts are used. As the dc current path to the base terminal is ignored $Q_{B}$ may be written

$$
Q_{B}(\varphi)=Q_{B 0}+Q_{B 1} \cos \left(\varphi-\theta_{q}\right) .
$$

The mean base charge $Q_{B 0}$ may be thought of as being injected during the initial transients of the amplifier.

According to Fig. 2, the transistor terminal voltage components may be broken up into the terms

$$
\begin{aligned}
& \left.\begin{array}{l}
V_{b 0}=V_{E 0} \\
V_{t 0}=V_{E 0}+V_{C 0}+V_{R 0}
\end{array}\right\} \text { mean values } \\
& \vec{V}_{b 1}=\vec{V}_{E L 1}+\vec{V}_{E 1}+\vec{V}_{B R 1}+\vec{V}_{B L 1} \quad \text { 1st harmonics } \\
& \left.\vec{V}_{t 1}=\vec{V}_{E L 1}+\vec{V}_{E 1}+\vec{V}_{C 1}+\vec{V}_{R 1}+\vec{V}_{C L 1}\right\} \text { (phasors). }
\end{aligned}
$$

Clearly, the contributions from linear elements need no further consideration. The terms from the nonlinear elements in Fig. 2, i.e., from $V_{E}, V_{C}$, and $V_{R}$ are considered below, with emphasis on how the nonlinearities are characterized in order to keep the calculations as simple as possible while maintaining the modeling accuracy. The final expressions for the terms in (9)-(12) are summarized in Appendix I.

\section{A. The Emitter Junction Voltage $V_{E}$}

An example of the charge-voltage relationship in a emitter junction is shown in Fig. 5. ${ }^{1}$ Within the voltage range from forward bias to the Zener breakdown level it is seen that if the charge drive $Q_{E}$ is sufficiently large and centered around zero, a piecewise-linear approximation is reasonable. This may both provide the foundations for calculating the $V_{E}$ components and also indicate the transitions between the cutoff and the conducting states of the transistor, i.e., cutoff:

$$
Q_{E}(\varphi) \leqq Q_{E 0} \Rightarrow\left\{\begin{array}{l}
V_{E}=Q_{E} / C_{E} \\
Q_{\mathrm{Ed}}=0
\end{array}\right.
$$

\footnotetext{
'The curves in Figs. 5 and 6 are calculated from conventional transistor modeling based on junction capacitance and transit time measurements [6].
} 


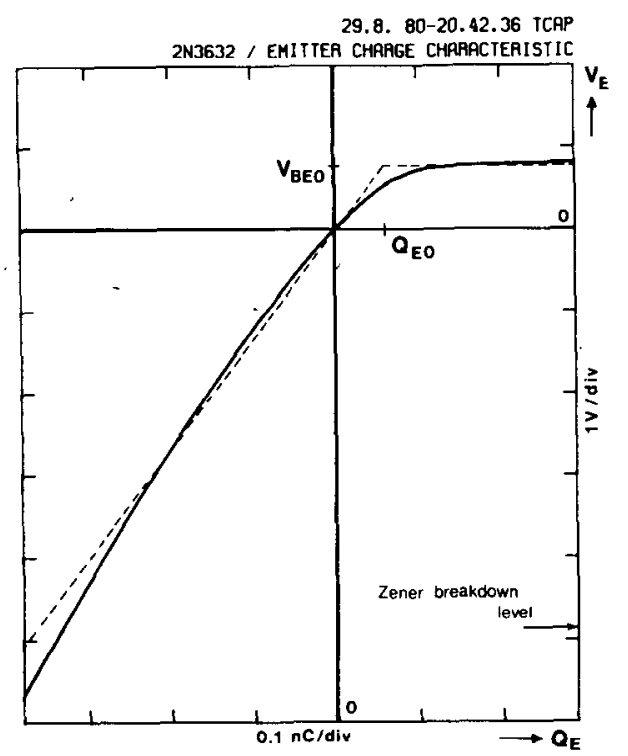

Fig. 5. Example of a detailed emitter capacitance characteristic. The dashed line shows its piecewise-linear approximation.

conduction:

$$
Q_{E}(\varphi)>Q_{E 0} \Rightarrow\left\{\begin{array}{l}
V_{E}=V_{\mathrm{BE} 0} \\
Q_{\mathrm{Ed}}=Q_{E}(\varphi)-Q_{E 0}
\end{array}\right.
$$

Herc $C_{E}$ denotes the linearized emitter junction capacitance, and $Q_{\mathrm{Ed}}$ denotes the emitter diffusion charge. Using (7) and the fact that during the cutoff period the total collector current is forced through the emitter capacitance, the emitter voltage may be written

$V_{E}(\varphi)=\left\{\begin{array}{c}V_{\mathrm{BE} 0}+\frac{Q_{B 1}}{C_{E}}\left[\cos \left(\varphi-\theta_{q}\right)-\cos \theta_{q}\right] \\ +\frac{Q_{E}(\varphi)-Q_{C 0}}{C_{E}}, \quad 0 \leqslant \varphi \leqslant \theta_{a} \\ V_{\mathrm{BE} 0}, \quad \theta_{a} \leqslant \varphi \leqslant 2 \pi\end{array}\right.$

where $Q_{C}(\varphi)$ is found by integration of (6) to yield

$$
Q_{C}(\varphi)=Q_{C 0}+\frac{I_{C 0}}{\omega_{g}} \varphi+\frac{I_{C 1}}{\omega_{g}}\left[\sin \left(\varphi-\theta_{C}\right)+\sin \theta_{C}\right] .
$$

The quantity $Q_{C 0}$ is the collector charge at the entrance to the cutoff state which is given by (24) below. From (17) the components in (9)-(12) provided by the emitter voltage are easily calculated to give the results in Appendix I.

\section{B. The Collector Junction Voltage $V_{C}$}

It is seen if Fig. 4 that the major contribution to the collector junction voltage pulse appears during the cutoff period which, therefore, requires detailed consideration. Less significant is the closing of the pulse during the forward active state of the transistor and a more crude description may be used here. The obvious choice is a straight line approximation to the trailing edge.

$\mathrm{RF}$-power transistors are commonly designed so that the collector voltage swing at the rated operation covers the greater part of the interval between zero and the avalanche level. The detailed collector characteristic in Fig: 6, there-

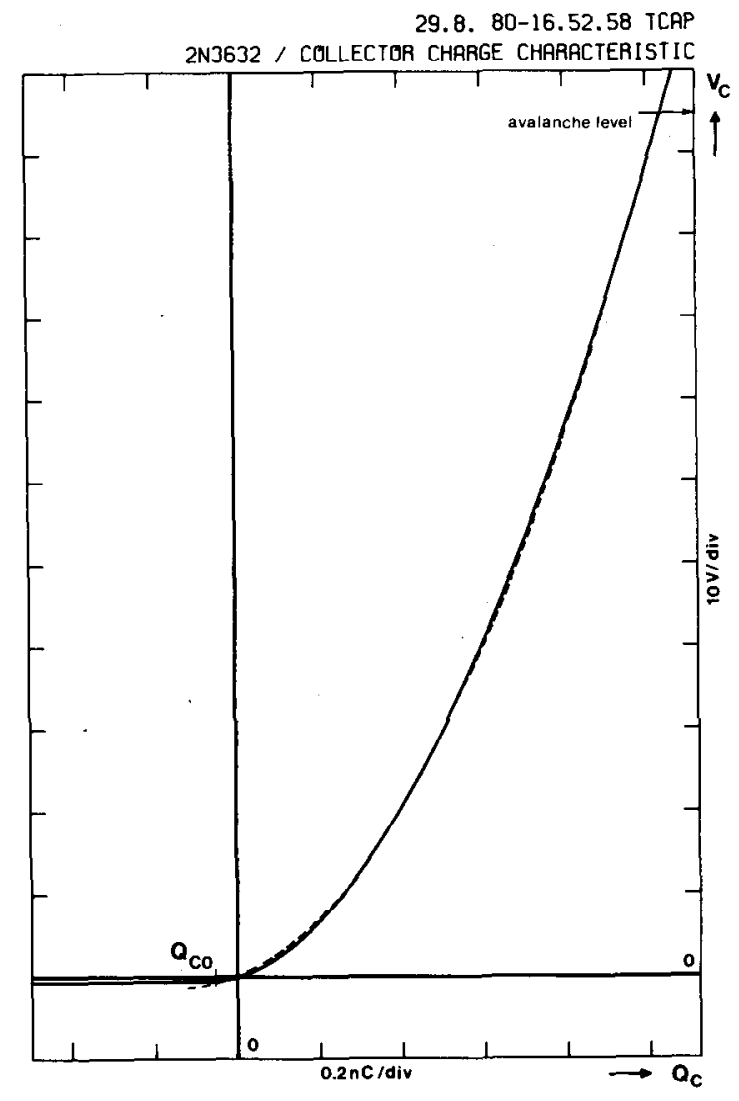

Fig. 6. Example of a detailed collector capacitance characteristic. The dashed line shows its parabolic approximation.

fore, demonstrates that a simple piecewise-linear approach does not suffice in this case. On the other hand, the charge voltage characteristic which results from an integration of the normal junction capacitance expression,

$$
\begin{aligned}
C_{j}\left(V_{C}\right) & =\frac{C_{0}}{\left(1+V_{C} / \Phi_{c}\right)^{n}} \Rightarrow \\
V_{C} & =\Phi_{C}\left[\left\{1+\frac{(1-n) Q_{C}}{C_{0} \Phi_{C}}\right\}^{1 /(1-n)}-1\right]
\end{aligned}
$$

does not invite for a calculation of the Fourier coefficients on the basis of the charge function in (18). It is demonstrated in Fig. 6 that a parabolic approximation in the form

$$
V_{C}=\alpha Q_{C}^{2}+\beta Q_{C}
$$

gives a good fit to the charge-voltage relationship and this will be used. As common measurement techniques identify the parameters in (19) or (20), the coefficients $\alpha$ and $\beta$ may be found by forcing the two expressions (20) and (21) to coincide at the rated collector supply and the avalanche level.

Using (18) and (21) the collector voltage in excess of $-\Phi_{C}$ during the cutoff period may be written

$$
V_{C x}(\hat{\varphi})=A+B \hat{\varphi}+C \hat{\varphi}^{2}+D \sin \hat{\varphi}+E \sin ^{2} \hat{\varphi}+F \hat{\varphi} \sin \hat{\varphi}
$$

where

$$
\hat{\varphi}=\varphi-\theta_{C}
$$


and

$$
\begin{array}{rlrl}
A & =\alpha Q_{i}^{2}+\beta Q_{i}+\Phi_{C} & D & =2 \alpha Q_{i} Q_{1}+\beta Q_{1} \\
B & =2 \alpha Q_{i} Q_{0}+\beta Q_{0} & E & =\alpha Q_{1}^{2} \\
C & =\alpha Q_{0}^{2} & F & =2 \alpha Q_{0} Q_{1} \\
Q_{0} & =I_{C 0} / \omega_{g} & Q_{1} & =I_{C 1} / \omega_{g} \\
Q_{i} & =I_{C 0} \theta_{C} / \omega_{g}+I_{C 1} \sin \theta_{C} / \omega_{g}+Q_{C 0} .
\end{array}
$$

The initial charge $Q_{C 0}$ corresponds to the collapse of the built-in potential and thereby (21) gives

$$
Q_{C 0}=-\frac{\beta}{2 \alpha}\left(1-\sqrt{1-4 \alpha \Phi_{C} / \beta^{2}}\right)
$$

At the onset of the active period the charge and the voltage are denoted,

$$
Q_{C T}=Q_{C}\left(\theta_{a}\right) \quad V_{C T}=\alpha Q_{C T}^{2}+\beta Q_{C T}
$$

and then the complete voltage wave shape becomes

$$
V_{C}(\varphi)=\left\{\begin{array}{lc}
-\Phi_{C}+V_{C x}\left(\varphi-\theta_{C}\right), & 0 \leqslant \varphi \leqslant \theta_{a} \\
-\Phi_{C}+\left(V_{C T}+\Phi_{C}\right) \frac{\theta_{\mathrm{bw}}-\varphi}{\theta_{\mathrm{bw}}-\theta_{a},} & \theta_{a} \leqslant \varphi \leqslant \theta_{\mathrm{bw}} \\
-\Phi_{C}, & \theta_{\mathrm{bw}} \leqslant \varphi \leqslant 2 \pi .
\end{array}\right.
$$

From this form the pertinent mean-value and first harmonics voltage components may be found from straightforward Fourier coefficient calculations.

\section{$C$. The Voltage Across the Collector Series Resistance $V_{R}$}

The series voltage is most conveniently expressed as the difference between the saturation characteristic $V_{\mathrm{RS}}$ and a base widening induced correction $V_{\mathrm{RBW}}$. According to (1) this may be written

$$
\begin{aligned}
V_{R}(\varphi) & =V_{\mathrm{RS}}(\varphi)-V_{\mathrm{RBW}}(\varphi) \\
& = \begin{cases}V_{\mathrm{RS}}(\varphi)-0, & 0 \leqq \varphi \leqq \theta_{\mathrm{bw}} \\
V_{\mathrm{RS}}(\varphi)-\eta(\varphi) V_{\mathrm{RS}}(\varphi), & \theta_{\mathrm{bw}} \leqq \varphi \leqq 2 \pi\end{cases}
\end{aligned}
$$

The saturation characteristic in (2) does not allow derivations of the Fourier coefficients in analytic forms. However, an expression showing the same asymptotic properties of ohmic conduction and saturation is

$$
V_{\mathrm{RS}}=R_{C 0} I_{\lim } \frac{I_{C} / I_{\lim }}{1-\left(I_{C} / I_{\lim }\right)^{2}} \text {. }
$$

This may be used without significant changes from the established set of empirical saturation expressions as the parameters in any case have to be fitted to the particular formula. Using (6) the characteristic can be written

$$
\begin{aligned}
& V_{\mathrm{RS}}(\varphi)=\frac{R_{C 0} I_{\lim }^{2}}{2 I_{C 1}}\left[\frac{1}{(\delta-\zeta)-\cos \left(\varphi-\theta_{C}\right)}\right.\left.-\frac{1}{(\delta+\zeta)+\cos \left(\varphi-\theta_{C}\right)}\right], \\
& \quad \delta=I_{\lim } / I_{C 1}, \quad \zeta=I_{C 0} / I_{C 1} .
\end{aligned}
$$

Thereby the mean-value and the first harmonic components of $V_{\mathrm{RS}}$ may be expressed by standard functions.

Using (4), (16), (7), and (27) the base widening caused correction becomes

$$
\begin{aligned}
& V_{\mathrm{RBW}}(\varphi)= \\
& {\left[\sqrt{\frac{Q_{B 1} \cos \left(\varphi-\theta_{q}\right)+Q_{B 0}+Q_{C 0}-Q_{E 0}}{\tau_{x}\left[I_{C 0}+I_{C 1} \cos \left(\varphi-\theta_{C}\right)\right]}}-\sqrt{\frac{\tau_{f 0}}{\tau_{x}}}\right] V_{\mathrm{RS}}(\varphi),} \\
& 0 \leqq V_{\mathrm{RBW}} \leqq V_{\mathrm{RS}}, \quad \theta_{\mathrm{bw}} \leqq \varphi \leqq 2 \pi . \quad \text { (30) }
\end{aligned}
$$

This expression fulfils the requirements for working out the corresponding Fourier coefficients in terms of elliptic integrals. In order to find their values, however, numerical integration methods must be employed. Therefore it makes more sense to use numerical integrations in conjunction with (30) directly for evaluating the mean-value and the first harmonics of $V_{\mathrm{RBW}}(\varphi)$. This approach has been chosen.

\section{COMPLETION OF THE AMPLIFIER MODEL}

In its final form the amplifier model is represented by a system of algebraic equations

$$
\boldsymbol{F}\left(\boldsymbol{x} \mid \boldsymbol{p}_{\text {opr }}, \boldsymbol{p}_{\text {trans }}^{\text {ref }}\right)=\mathbf{0}
$$

which must be solved numerically for the unknown $x$. The parameters in (31) are the operational conditions $p_{\text {opr }}$ and the transistor parameters $\boldsymbol{p}_{\text {trans }}^{\text {ref }}$. These refer to a specific transistor chip temperature $T_{\text {ref }}$. The actual transistor parameters to be used at a chip temperature which differs from the references by $\Delta T$ have the general relationship

$$
\left.p_{\text {trans }}\right|_{T=\Delta T+T_{\text {rct }}}=p_{\text {trans }}\left(\Delta T, p_{\text {trans }}^{\text {ref }}\right) \text {. }
$$

A summary of the transistor parameters and their thermal dependencies is given in Appendix II.

The variables introduced so far for characterizing the operation of the transistor constitute the unknowns of the model, i.e.,

$$
x=\left\{I_{C 0}, I_{C 1}, \theta_{C}, Q_{B 0}, Q_{B 1}, \theta_{q}, \theta_{a}, \theta_{\mathrm{bw}}, \Delta T\right\} .
$$

In short form the findings of the preceding section may. thereby be written

$$
\begin{aligned}
V_{b 0}, V_{t 0}, Q_{C T} & =V_{b 0}, V_{t 0}, Q_{C T}\left(x \mid \omega_{g}, p_{\text {trans }}^{\text {ref }}\right) \\
\vec{V}_{b 1}, \vec{V}_{t 1} & =\vec{V}_{b 1}, \vec{V}_{t 1}\left(x \mid \omega_{g}, p_{\text {trans }}^{\text {ref }}\right) .
\end{aligned}
$$

The actual choice of the operation conditions $p_{\text {opr }}$ depends on how the amplifier is configured, biased, loaded, and driven. This also applies to the system of equations in (31) which accordingly may be subdivided into

$$
\boldsymbol{F}=\left\{\boldsymbol{F}_{\text {basic }}, \boldsymbol{F}_{\text {bias }}, \boldsymbol{F}_{\text {load }}, \boldsymbol{F}_{\text {drive }}\right\}^{t}=\mathbf{0} .
$$

Here $\boldsymbol{F}_{\text {basic }}$ represents the subsystem of equations which is connected to the operation of the transistor and which is in common for all applications. This includes four equations which appear as follows.

The charge balance in the base is by the approximations in (13)-(16) completely fixed at the instants $\varphi=0$ and 
$\varphi=\theta_{a}$. Using (7), (8), and (25) this gives

$$
\begin{array}{r}
Q_{B 1} \cos \theta_{q}+Q_{B 0}+Q_{C 0}-Q_{E 0}=0 \\
Q_{B 1} \cos \left(\theta_{a}-\theta_{q}\right)+Q_{B 0}+Q_{C T}-Q_{E 0}=0 .
\end{array}
$$

During the active period the transistor switching is governed by

$$
\frac{d Q_{C}}{d \varphi}+\frac{Q_{C}}{\epsilon}=\frac{1}{\epsilon}\left[\tau_{f 0} I_{C}(\varphi)-Q_{B}(\varphi)+Q_{E 0}\right]
$$

where $\epsilon=\omega_{g} \tau_{f 0}$. The equation may be found from Fig. 2, applying the current law to the intrinsic collector node and using (7) and (16). With the initial condition $Q_{C}\left(\theta_{a}\right)=Q_{C T}$ and the requirement $Q_{C}\left(\theta_{\mathrm{bw}}\right)=Q_{\mathrm{C} 0}$, the solution to (39) provides

$$
\begin{aligned}
\frac{1}{\sqrt{1+\epsilon^{2}}}\left[\tau_{f 0} I_{C 1} \cos \left(\theta_{\mathrm{bw}}-\theta_{C}-\theta_{\mathrm{\epsilon}}\right)-Q_{B 1} \cos \left(\theta_{\mathrm{bw}}-\theta_{q}-\theta_{\epsilon}\right)\right] \\
+\left[Q_{C T}+\frac{1}{\sqrt{1+\epsilon^{2}}}\left\{Q_{B 1} \cos \left(\theta_{a}-\theta_{q}-\theta_{\epsilon}\right)\right.\right. \\
\left.\left.-\tau_{f 0} I_{C 1} \cos \left(\theta_{a}-\theta_{C}-\theta_{\epsilon}\right)\right\}\right] \cdot E \\
+\left(\tau_{f 0} I_{C 0}-Q_{B 0}+Q_{E 0}\right)(1-E)-Q_{C 0}=0
\end{aligned}
$$

where

$$
\theta_{\epsilon}=\tan ^{-1} \epsilon \text { and } E=\exp \left[\left(\theta_{a}-\theta_{b w}\right) / \epsilon\right] .
$$

Finally the temperature balance may be found from the total power dissipation in the transistor

$\Delta T+T_{\mathrm{ref}}-T_{\mathrm{amb}}-R_{\mathrm{th}}\left\{V_{t 0} I_{C 0}+\frac{1}{2} \operatorname{Re}\left[\vec{V}_{b 1} \vec{I}_{B 1}^{*}+\vec{V}_{t 1} \vec{I}_{C 1}^{*}\right]\right\}=0$

where $T_{\mathrm{amb}}$ is the ambient temperature, $R_{\mathrm{th}}$ is the total thermal resistance of the transistor, and $I_{B 1}$ denotes the base current

$$
\vec{I}_{B 1} \sim \omega_{g} Q_{B 1} \cos \left(\varphi-\theta_{q}+\frac{\pi}{2}\right) .
$$

One of the simplest methods of operating and biasing the transistor is shown by the equivalent circuit in Fig. 7. This will be used below as an example of a complete amplifier model. Here the remaining equations become

$$
\begin{aligned}
& \boldsymbol{F}_{\text {bias }}=\mathbf{0}:\left\{\begin{array}{l}
V_{b 0}-V_{B B}=0 \\
V_{t 0}-V_{C C}=0
\end{array}\right. \\
& \boldsymbol{F}_{\text {load }}=\mathbf{0}: \vec{V}_{t 1}+Z_{L} \vec{I}_{C 1}=0 \\
& \boldsymbol{F}_{\text {drive }}=\mathbf{0}:\left|V_{b 1}\right|^{2}+\left|Z_{g} I_{B 1}\right|^{2}+2 \operatorname{Re}\left\{\vec{V}_{B 1} Z_{g}^{*} \vec{I}_{B 1}^{*}\right\}-\left|V_{g}\right|^{2}=0 .
\end{aligned}
$$

Including the basic equations the number of constraints now corresponds to the number of unknowns so the model is in principle ready for solution with the operational conditions:

$$
p_{\text {opr }}=\left\{\omega_{g}, V_{g}, R_{g}, X_{g}, R_{L}, X_{L}, V_{C C}, V_{B B}, T_{\text {amb }}\right\} .
$$

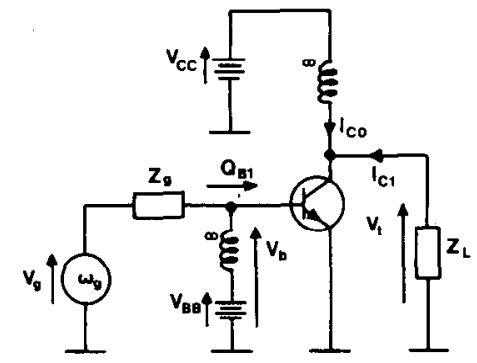

Fig. 7. Simple equivalent diagram for completing the amplifier model. The chokes are assumed infinitely large. Their actual impedances may be included in $Z_{g}$ and $Z_{L}$.

In practice a series of additional aspects remain to be considered, for instance, simplifications of the system of equations through variable eliminations and transformations, methods for initializing, and the problem of keeping the numerical solution process of the trace. However, these are beyond the scope of this presentation. More details may be found in [7] where also a series of other methods for biasing and operating the transistor are discussed.

\section{Verifications of the Describing FunCtion METHOD}

Having found a solution to (31) a long row of derived quantities like output power, transistor impedances, efficiency, etc., may be calculated either directly from the results or by adding more details about the matching network functions as those required for setting up Fig. 7. In order to demonstrate the practical capabilities of the describing function approach, some examples of calculated and experimental performances for the amplifier in Fig. 8 will be considered.

Fig. 9 shows the output power, the collector efficiency and the input port reflected voltage as functions of the drive level in the case where the circuit is left unchanged after tuning. The reflection may be calculated from the difference between the available and the consumed power at the base interface and gives a measure for the ability of the model to reproduce the transistor input impedance variations. As seen, the agreement between computations and measurements is good at higher drive levels, whereas discrepancies occur at low levels. Time-domain simulations have shown that here the reason is that the sharp distinction between the active and the base widening controlled mode of operation which underlie the approximated modeling is no longer valid [7]. Thereby (3) becomes inaccurate and, as the drive level is reduced, the accuracy of the piecewise-linear emitter characteristic eventually ceases.

Very little would be gained if the results from the describing function method need time-domain simulation backup before their usefulness is secured. Therefore, a series of indicator functions showing whether or not the modeling assumptions of the transistor are violated have been incorporated in the computations. In the above example the ignorance of the active period which follows. the base widening period gives the indication. If the ignorance is legal it may be shown [7] that the solution function to 


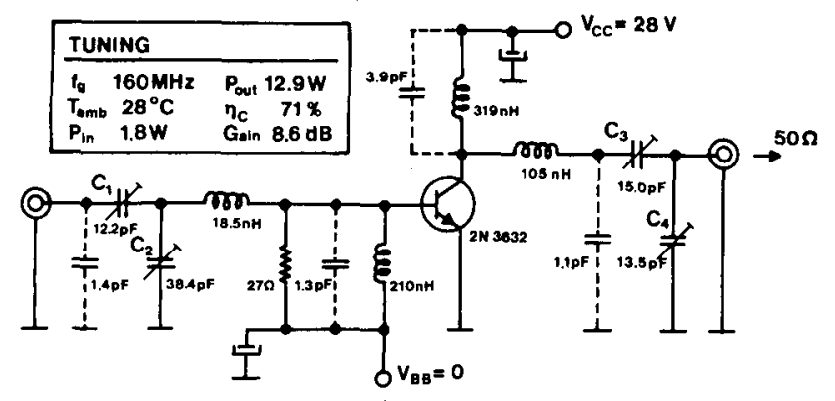

Fig. 8. Full equivalent circuits for the matching networks of the RFpower amplifier under test. The transistor equivalent is given by Fig. 2 and Table I.

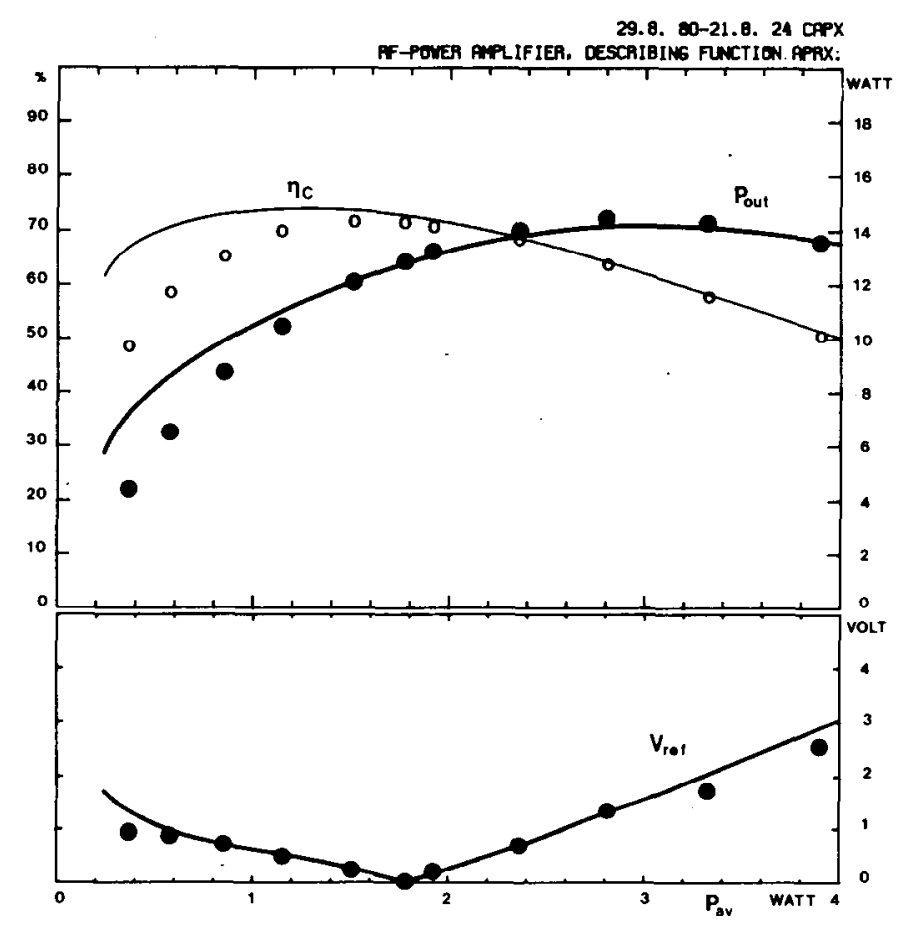

Fig. 9. Experimental (dots) and calculated amplifier output power, collector efficiency, and input port reflected voltage as functions of the available power. The discrepancies at low drive levels are caused by violations of the modeling assumptions.

(39), which was used for setting up the condition in (40), at the instant $\varphi=2 \pi$ should give a charge which is less than or approximates $Q_{C 0}$, i.e., the small, negative amount which applies during the base widening period. As demonstrated by Fig. 10, the sign of this stipulated solution which, taken relative to $Q_{C 0}$, is denoted $q_{C 0}$ gives a good bound for the applicability of the simulated results. Moreover, the figure also demonstrates the connection between the disappearance of the base widening and the violation of the modeling assumptions.

The ability of the proposed method to cope with situations where the collector load departs from its nominal value is demonstrated by Fig. 11 . The adjustment range for the trimmer $\mathrm{C}_{3}$ corresponds to an imaginary part impedance variation between -45 and $67 \Omega$, while the real part remains nearly constant at $30 \Omega$. Also in this case basic assumptions are violated although the consequences are not so severe as above. Here it is the ignorance of the dc

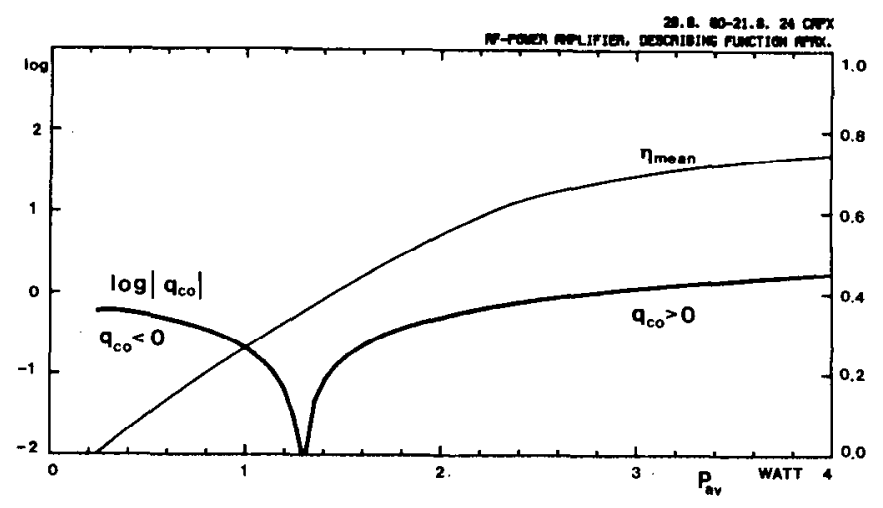

Fig. 10. Progress of the modeling consistency indicator function $q_{C 0}$ corresponding to the example in Fig. 9. Consistency is assured on positive values. $\eta_{\text {mean }}$ denotes the calculated mean-value of the base widening ratio over the base widening period.

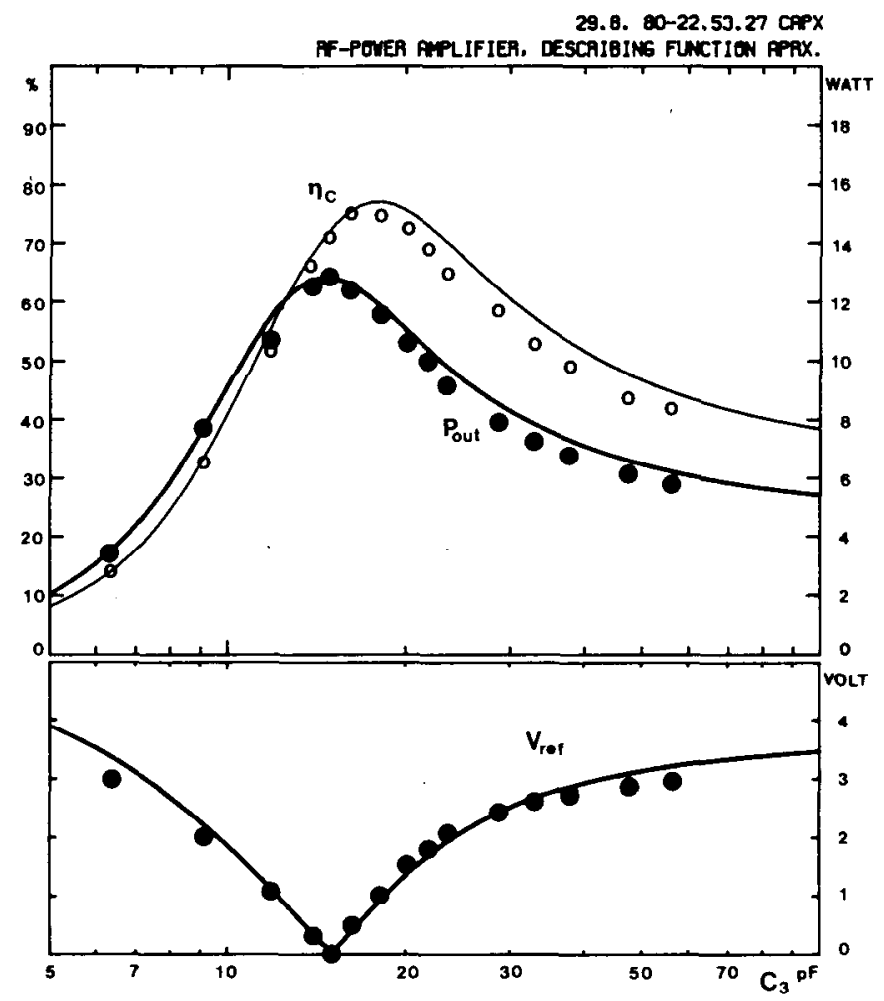

Fig. 11. Experimental (dots) and calculated amplifier output power, collector efficiency, and input reflection as functions of the collector tuning.

currents in the base, and the indicator function showing this is the peak-value of the collector junction voltage. It may be calculated from (22) using the instant when the collector current crosses zero downwards, if this happens in the active period, by setting $V_{C \max }=V_{C T}$ from (25). As shown in Fig. 12, $V_{C \max }$ exceeds the avalanche breakdown level if the load becomes too inductive. The figure also shows the transistor-chip temperature variations, and the importance of including thermal effects in the simulations may be understood from the fact that within this range, the linear series resistance $R_{C U}$ changes by a factor of more than three.

Finally, Fig. 13 gives the experimental and simulated frequency response of the test amplifier. The computed 


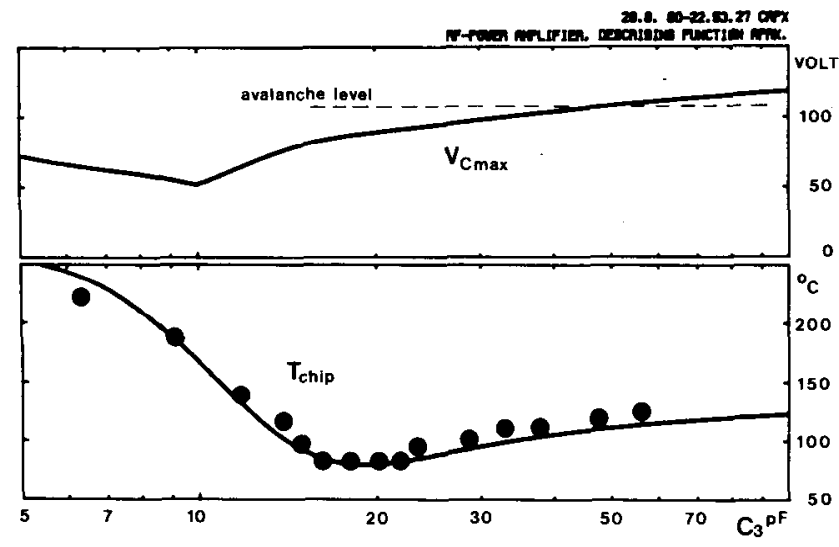

Fig. 12. Calculated maximum collector junction voltage and chip temperature corresponding to the example in Fig. 12. The experimental chip temperature (dots) are estimated from the case temperatures and the thermal resistances of the transistor and the heat sink.

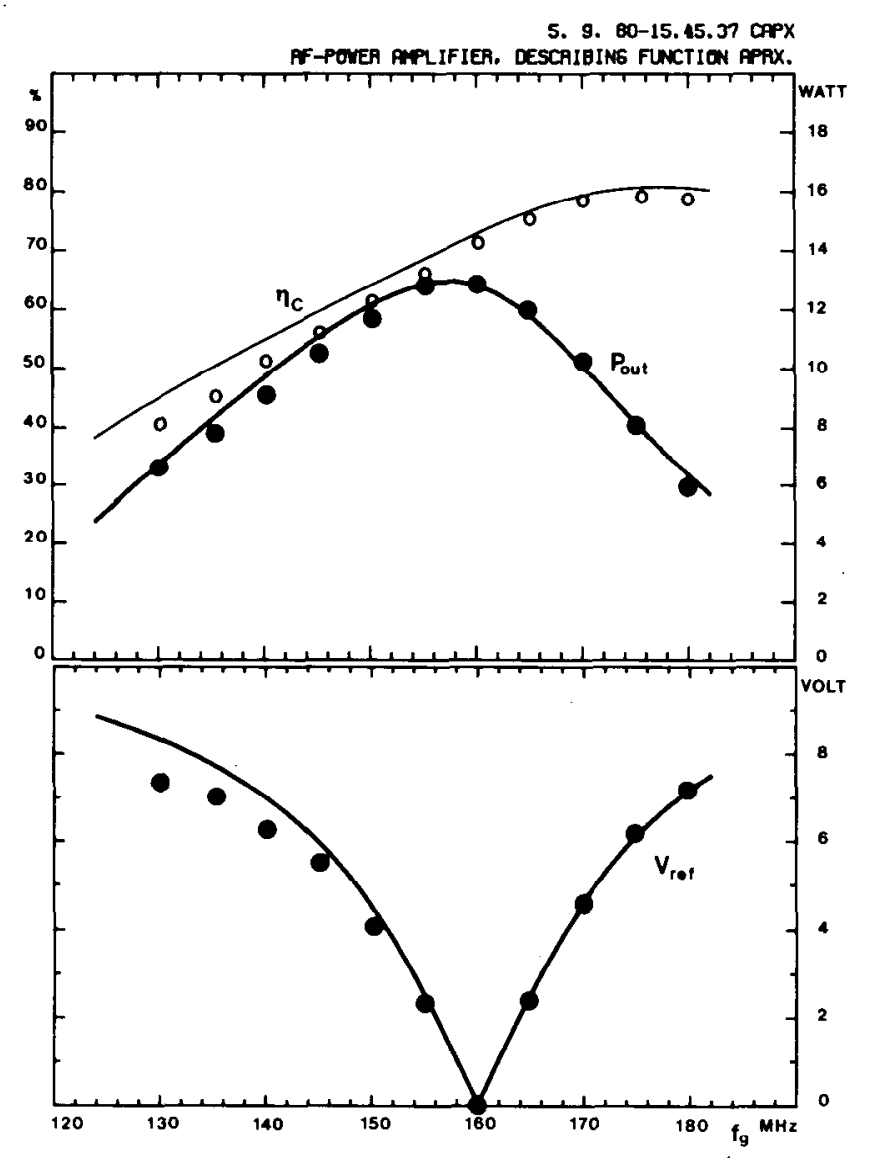

Fig. 13. Experimental (dots) and calculated frequency responses of output power, collector efficiency and input reflection.

charactcristics are shown in the whole interval where it is possible to get convergency of the solution to (31). What happens outside this interval is unexplored, but the useful range covers more than the $3-\mathrm{dB}$ boundaries as seen.

\section{DISCUSSIONS AND CONCLUSIONS}

The above results demonstrate the ability of the describing function approach to provide useful results within its fundamental limitations, as stated by conditions A), B), and $\mathrm{C}$ ) in Section I. The speed whereby these results are found is best characterized by the fact that in average 700 solutions to (31) are possible within the same computing time as the one required for achieving a single steady-state operation cycle by the time-domain simulation in [2].

Considering the use of the proposed method, three areas are presently being investigated.

\section{1) Design Aid:}

In this respect it is especially important that the method calculates the input impedance of the transistor with reasonable accuracy. This quantity causes most troubles in design being both rather sensitive to operational conditions and difficult to measure. Data sheets give some information, but they often refer to a specific test circuit which has been optimally tuned. In broad-band designs this does not suffice as the optimum cannot apply over the full bandwidth. Used interactively or as a part of a design automation program, the method will provide the necessary parameters for synthesis of the matching or interstage coupling networks.

\section{2) Secondary Properties:}

Being basically a single-frequency steady-state analysis where the feedback effects in the circuit are laid down directly in the describing functions, the presented method cannot account for secondary properties such as distortion, intermodulation and stability. However, as the modeling is based on a physically oriented nonlinear transistor equivalent diagram, a solution may provide the necessary parameters for other types of analysis where such effects are considered. This is supposed to be especially useful in connection with methods using series expansions around an operation cycle.

3) Basic Insight:

The ideas using describing functions and switch equivalents to the transistor operation in high efficiency amplifiers are as old as the presence of such circuits. The principles of the class $\mathrm{B}, \mathrm{C}, \mathrm{D}$, and $\mathrm{E}$ modes of operations are commonly explored by applying these concepts on the output part of the circuit and then using the conducting angle or the switch duty cycle as the forcing function [8], [9]. This method is illustrative and may provide reasonable results if the transistor is operated far below its ratings with respect to frequency and power. Approaching the ratings, however, the conducting angle cannot be foreseen and, moreover, the distinctions between the different classes become blurred. In this case, there is a need for gaining more insight in problem areas as the selection of design objectives, and which circuit and transistor properties set the boundaries for optimum design. The strength of the new method described above is that it comprises the complete circuit from the input port to the load. It is, therefore, thought that it may be a useful tool for undertaking this task.

\section{APPENDIX I}

\section{Summary of Describing Functions}

All the terms in (9)-(12) which may be stated in analytic form are listed below. The first harmonics are given by their cosine and sine components. In cases where these, 
most conveniently, refer to another phase origin than the one used in the complete approximation problem, this is indicated by a superscript. The auxiliary origin must be compensated when the final phasor additions in (11), (12) are made.

A-1. Linear Contributions, $V_{E L}, V_{B L}, V_{B R}, V_{C L}$

$$
\begin{aligned}
& V_{E L 1, \cos }=\omega_{g} L_{E}\left[-\omega_{g} Q_{B 1} \cos \theta_{q}+I_{C 1} \sin \theta_{C}\right] \\
& V_{E L 1, \sin }=\omega_{g} L_{E}\left[\omega_{g} Q_{B 1} \sin \theta_{q}+I_{C 1} \cos \theta_{C}\right] \\
& V_{B L 1, \cos }^{\theta_{q}}=\omega^{2} L_{B L} Q_{B 1} \quad V_{B L 1, \sin }^{\theta_{q}}=0 \\
& V_{B R 1, \cos }^{\theta_{q}}=0 \quad V_{B R 1, \sin }^{\theta_{g}}=-\omega_{g} Q_{B 1} R_{B} \\
& V_{C L 1, \cos }^{\theta_{C}}=0 \quad V_{C L 1, \sin }^{\theta_{C}}=-\omega_{g} I_{C 1} L_{C L} .
\end{aligned}
$$

\section{A-2. Emitter Junction Voltage $V_{E}$}

$$
\begin{aligned}
V_{E 0}= & V_{B E 0}+\frac{Q_{B 1}}{2 \pi C_{E}}\left[\sin \left(\theta_{a}-\theta_{q}\right)+\sin \theta_{q}-\theta_{a} \cos \theta_{q}\right] \\
& +\frac{1}{2 \pi C_{E} \omega_{g}}\left[I_{C 0} \frac{\theta_{a}^{2}}{2}+I_{C 1}\left(\theta_{a} \sin \theta_{C}+\cos \theta_{C}\right.\right. \\
& \left.\left.-\cos \left(\theta_{a}-\theta_{C}\right)\right)\right] \\
V_{E 1, \cos }= & \frac{Q_{B 1}}{\pi C_{E}}\left[\frac{\theta_{a}}{2} \cos \theta_{q}+\frac{1}{4}\left(\sin \left(2 \theta_{a}-\theta_{q}\right)+\sin \theta_{q}\right)\right. \\
& \left.-\cos \theta_{q} \sin \theta_{a}\right]+\frac{1}{\pi C_{E} \omega_{g}}\left[\frac { I _ { C 1 } } { 4 } \left(-2 \theta_{a} \sin \theta_{C}\right.\right. \\
& \left.-\cos \left(2 \theta_{a}-\theta_{C}\right)+\cos \theta_{C}+4 \sin \theta_{C} \sin \theta_{a}\right) \\
& \left.+I_{C 0}\left(\cos \theta_{a}+\theta_{a} \sin \theta_{a}-1\right)\right] \\
V_{E 1, \sin } & \frac{Q_{B 1}}{\pi C_{e}}\left[\frac{\theta_{a}}{2} \sin \theta_{q}-\frac{1}{4}\left(\cos \left(2 \theta_{a}-\theta_{q}\right)-\cos \theta_{q}\right)\right. \\
& \left.\left.+\cos \theta_{a}-1\right)\right]+\frac{1}{\pi C_{E} \omega_{g}}\left[\frac { I _ { C 1 } } { 4 } \left(2 \theta_{a} \cos \theta_{C}\right.\right. \\
& \left.-\sin \left(2 \theta_{a}-\theta_{C}\right)-4 \sin \theta_{C} \cos \theta_{a}+3 \sin \theta_{C}\right) \\
& \left.+I_{C 0}\left(\sin \theta_{a}-\theta_{a} \cos \theta_{a}\right)\right] .
\end{aligned}
$$

\section{$A$-3. Collector Junction Voltage $V_{C}$}

$$
\begin{aligned}
V_{C 0}= & -\Phi_{C}+\frac{A}{2 \pi}\left(\varphi_{u}-\varphi_{l}\right)+\frac{B}{4 \pi}\left(\varphi_{u}^{2}-\varphi_{l}^{2}\right)+\frac{C}{6 \pi}\left(\varphi_{u}^{3}-\varphi_{l}^{3}\right) \\
& +\frac{D}{2 \pi}\left(-\cos \varphi_{u}+\cos \varphi_{l}\right)+\frac{E}{4 \pi}\left[\left(\varphi_{u}-\varphi_{l}\right)\right. \\
& \left.-\frac{1}{2}\left(\sin 2 \varphi_{u}-\sin 2 \varphi_{l}\right)\right] \\
& +\frac{F}{2 \pi}\left[\sin \varphi_{u}-\sin \varphi_{l}-\varphi_{u} \cos \varphi_{u}\right. \\
& \left.+\varphi_{l} \cos \varphi_{l}\right]+\frac{1}{4 \pi}\left(V_{C T}+\Phi_{C}\right)\left(\theta_{\mathrm{bw}}-\theta_{a}\right)
\end{aligned}
$$

where

$$
\varphi_{u}=\theta_{a}-\theta_{C} \quad \varphi_{l}=-\theta_{C} .
$$

The first harmonics may be broken up according to

$$
\vec{V}_{C l}=\vec{V}_{C 01}+\vec{V}_{a 1}
$$

where the two terms originate from the cutoff and the active period, respectively, cf., (26). These have the components

$$
\begin{aligned}
V_{C 01, \cos }^{\theta_{C}=} & \frac{A}{\pi}\left[\sin \varphi_{u}-\sin \varphi_{l}\right]+\frac{B}{\pi}\left[\cos \varphi_{u}-\cos \varphi_{l}+\varphi_{u} \sin \varphi_{u}\right. \\
& \left.-\varphi_{l} \sin \varphi_{l}\right]+\frac{C}{\pi}\left[2\left(\varphi_{u} \cos \varphi_{u}-\varphi_{l} \cos \varphi_{l}\right)\right. \\
& \left.+\left(\varphi_{u}^{2}-2\right) \sin \varphi_{u}-\left(\varphi_{l}^{2}-2\right) \sin \varphi_{l}\right]+\frac{D}{2 \pi}\left[\sin ^{2} \varphi_{u}\right. \\
& \left.-\sin ^{2} \varphi_{l}\right]+\frac{E}{3 \pi}\left[\sin ^{3} \varphi_{u}-\sin ^{3} \varphi_{l}\right]+\frac{F}{2 \pi} \\
& \cdot\left[\frac{1}{2}\left(\sin \varphi_{u} \cos \varphi_{u}-\sin \varphi_{l} \cos \varphi_{l}\right)+\varphi_{u} \sin ^{2} \varphi_{u}\right. \\
& \left.-\varphi_{l} \sin \varphi_{l}^{2}-\frac{1}{2}\left(\varphi_{u}-\varphi_{l}\right)\right] \\
V_{C 01, \sin }^{\theta_{C}}= & \frac{A}{\pi}\left[-\cos \varphi_{u}+\cos \varphi_{l}\right]+\frac{B}{\pi}\left[\sin \varphi_{u}-\sin \varphi_{l}\right. \\
& \left.-\varphi_{u} \cos \varphi_{u}+\varphi_{l} \cos \varphi_{l}\right]+\frac{C}{\pi}\left[2\left(\varphi_{u} \sin \varphi_{u}-\varphi_{l} \sin \varphi_{l}\right)\right. \\
& \left.-\left(\varphi_{u}^{2}-2\right) \cos \varphi_{u}+\left(\varphi_{l}^{2}-2\right) \cos \varphi_{l}\right]+\frac{D}{2 \pi}\left[\varphi_{u}-\varphi_{l}\right. \\
& \left.-\frac{1}{2}\left(\sin 2 \varphi_{u}-\sin 2 \varphi_{l}\right)\right]+\frac{E}{\pi}\left[\frac{1}{3}\left(\cos \varphi^{3}-\cos ^{3} \varphi_{l}\right)\right. \\
& \left.-\cos \varphi_{u}+\cos \varphi_{l}\right]+\frac{F}{2 \pi}\left[\frac{1}{2}\left(\sin \varphi_{u}^{2}-\sin ^{2} \varphi_{l}\right)\right. \\
V_{a 1, \sin }= & \frac{1}{\pi}\left(V_{C T}+\Phi_{C}\right)\left[\frac{\sin \theta_{a}-\sin \theta_{\mathrm{bw}}}{\theta_{\mathrm{bw}}-\theta_{a}}+\cos \theta_{a}\right] . \\
& \left.-\varphi_{u} \cos \varphi_{u} \sin \varphi_{u}+\varphi_{l} \cos \varphi_{l} \sin \varphi_{l}+\frac{1}{2}\left(\varphi_{u}^{2}-\varphi_{l}^{2}\right)\right] \\
V_{a 1, \cos } & \frac{1}{\pi}\left(V_{C T}+\Phi_{C}\right)\left[\frac{\cos \theta_{a}-\cos \theta_{\mathrm{bw}}}{\theta_{\mathrm{bw}}-\theta_{a}} \sin _{a}\right] \\
&
\end{aligned}
$$

\section{$A$-4. Voltage Across the Collector Series Resistance $V_{R}$}

According to Section III-C $V_{R}$ is given by

$$
V_{R}=V_{\mathrm{RS}}-V_{\mathrm{RBW}}
$$

where the contributions from $V_{\mathrm{RBW}}$ are found by numerical integrations. The components of $V_{\mathrm{RS}}$ are

$$
\begin{gathered}
V_{\mathrm{RS} 0}=R_{C 0} I_{1 \mathrm{im}} \frac{\delta}{2}\left[\frac{1}{\sqrt{(\delta-\zeta)^{2}-1}}-\frac{1}{\sqrt{(\delta+\zeta)^{2}-1}}\right] \\
V_{\mathrm{RS} 1, \cos }^{\theta_{C}}=R_{C 0} I_{1 \mathrm{im}} \delta\left[\frac{(\delta-\zeta)}{\sqrt{(\delta-\zeta)^{2}-1}}+\frac{(\delta+\zeta)}{\sqrt{(\delta+\zeta)^{2}-1}}-2\right]
\end{gathered}
$$

$V_{\mathrm{RS}, \text { sin }}^{\theta_{c}}=0$. 
TABLE I

Transistor Parameters. (Superscript ref Indicates That the QUANTITY REFERS TO $T_{\text {ref }}$ )

\begin{tabular}{|c|c|c|}
\hline Parameter & Explanation & $\begin{array}{l}\text { Example } \\
2 \mathrm{~N} 3632\end{array}$ \\
\hline$v_{\mathrm{BEO}}^{r e f}$ & Emitter turn-on voltage & $0.78 \mathrm{~V}$ \\
\hline$c_{E}$ & Linearized emitter capacitance & $75.0 \mathrm{pF}$ \\
\hline$c_{0}^{r e f}$ & Zero-bias collector capacitance & $67.1 \mathrm{pF}$ \\
\hline$\phi_{C}^{\text {ref }}$ & Collector built-in potential & $0.48 \mathrm{~V}$ \\
\hline$n$ & Collector junction profile constant & 0.46 \\
\hline$v_{C C R}$ & Rated collector supply voltage & $28 \mathrm{~V}$ \\
\hline${ }^{B V}{ }_{C B O}$ & Collector avalanche voltage & $110 \mathrm{~V}$ \\
\hline$R_{\mathrm{CO}}^{\text {ref }}$ & Collector low-current series resistance & $4.21 \Omega$ \\
\hline$I_{\lim }^{\text {ref }}$ & Collector region scattering limited current & $2.80 \mathrm{~A}$ \\
\hline$\tau_{\text {fo }}$ & Forward transit time prior to basewidening & $0.15 \mathrm{~ns}$ \\
\hline$\tau_{\max }$ & Forward transit time at full basewidening & $1.60 \mathrm{~ns}$ \\
\hline $\mathrm{L}_{E}$ & Emitter lead inductance & $3.35 \mathrm{nH}$ \\
\hline $\mathrm{L}_{\mathrm{BL}}$ & Base lead inductance & $2.30 \mathrm{nH}$ \\
\hline $\mathrm{L}_{\mathrm{CL}}$ & Collector lead inductance & $3.0 \mathrm{nH}$ \\
\hline$R_{B}$ & Linearlzed base series resistance & $0.2 \Omega$ \\
\hline $\mathrm{T}_{\text {ref }}$ & Reference chip temperature & $25^{\circ} \mathrm{C}$ \\
\hline k & Thermal coefficient for $V_{B E O}$ & $-2.0 \mathrm{mV} /{ }^{\circ} \mathrm{C}$ \\
\hline $\mathrm{H}$ & Thermal coefficient for ${ }^{\mathrm{C}}$ & $-1.9 \mathrm{mV} /{ }^{\circ} \mathrm{C}$ \\
\hline Y & Thermal parameter for $\mathrm{R}_{\mathrm{co}}$ & 2.0 \\
\hline$T_{\theta}$ & Thermal parameter for $I_{1 \text { im }}$ & $600^{\circ} \mathrm{K}$ \\
\hline c & Thermal parameter for $I_{1 i m}$ & 0.8 \\
\hline$R_{\text {th }}$ & Total thermal resistance for transistor & $9.1^{\circ} \mathrm{C} / \mathrm{W}$ \\
\hline
\end{tabular}

\section{APPENDIX II}

\section{Summary of Transistor Parameters}

The complete list of all the transistor parameters required in the describing function characterization is shown in Table I. The most significant temperature effects to be taken into account are in priority the thermal variations of

1) The collector series resistance voltage $V_{R}$.

2) The collector capacitance $C_{j}$.

3) The emitter turn-on voltage $V_{\mathrm{BEO}}$.

This may be done by including the following relationships which are deducted from basic properties [10], [11]:

$$
\begin{aligned}
R_{C 0} & =R_{C 0}^{\mathrm{ref}}\left(1+\Delta T / T_{\mathrm{ref}}\left[{ }^{\circ} \mathrm{K}\right]\right)^{\gamma} \\
I_{\mathrm{lim}} & =I_{\lim }^{\mathrm{ref}} \frac{1+C \exp \left[T_{\mathrm{ref}}\left[{ }^{\circ} \mathrm{K}\right] / T_{\theta}\right]}{1+C \exp \left[\left(T_{\mathrm{ref}}\left[{ }^{\circ} \mathrm{K}\right]+\Delta T\right) / T_{\theta}\right]}
\end{aligned}
$$

$$
\begin{aligned}
\Phi_{C} & =\Phi_{C}^{\mathrm{ref}}+H \Delta T \\
C_{0} & =C_{0}^{\mathrm{ref}}\left(1+H \Delta T / \Phi_{C}^{\mathrm{ref}}\right)^{-n} \\
V_{\mathrm{BE} 0} & =V_{\mathrm{BE} 0}^{\mathrm{ref}}+K \Delta T .
\end{aligned}
$$

The thermal effects are carried over the coefficients $\alpha$ and $\beta$ through the fitting between (20) and (21) at $V_{C}=V_{C C R}$ and $V_{C}=B V_{C B 0}$.

The problem of assigning values to the parameters should not be underestimated. However, this is not a prime concern here so it shall only be mentioned that starting from scratch, the parameters

$$
R_{C 0}^{\mathrm{ref}}, I_{\mathrm{lim}}^{\mathrm{ref}}, \tau_{f 0}, \tau_{\max }, \gamma, L_{E}, L_{B L}
$$

are the most troublesome. They combine the properties of being both hard to measure and rather influent on the result of the approximation method. The rest of the parameters are either easily measured, deductable from data sheets and other common sources, or without more significance than a reasonable guess may suffice.

\section{REFERENCES}

[1] "MOS and Special Purpose Bipolar Integrated Circuits and RFPower Transistor Circuit Design," Texas Instr. Inc., NY, McGrawHill, 1976.

[2] J. Vidkjaer, "A computerized study of the class-c-biased RF-power amplifier," IEEE J. Solid-State Circuits, vol. SC-13, pp. 247-258, Apr. 1978

[3] D. L. Bowler and F. a. Lindholm, "High current regimes in transistor collector regions," IEEE Trans. Electron Devices, vol. ED-20, pp. 257-263, Mar. 1973.

[4] H. K. Gummel and H. C. Poon, "An integral charge control model of bipolar transistors," Bell System Tech. J., vol. 49, pp. 827-852, May 1970.

[5] R. Kumar and L. P. Hunter, "Prediction of $f_{T}$ and $h_{f e}$ at high collector currents," IEEE Trans. Electron Devices, vol. ED-22, pp. 1031-1037, Nov. 1975

[6] I. Getreu, "Modeling the Bipolar Transistor," Tektronix Inc., Beaverton, OR, 1976

[7] J. Vidkjaer, "Approximation methods for bipolar RF-power amplifier analysis," Electronics Inst. Rep. Tech. Univ., Denmark, to be published.

[8] F. H. Raab, "High efficiency amplification techniques," IEEE Circuits Syst., Newsletter, vol. 7, pp. 3-11, Dec. 1975.

[9] K. K. Clarke and D. T. Hess, Communication Circuits: Analysis and Design. Reading, MA, Addison-Wesley, 1971.

[10] C. Jacobini et al., " $\Lambda$ review of some charge transport propertics of silicon," Solid State Electron., vol. 20, pp. 77-89, Feb. 1977.

[11] S. K. Ghandi, Semiconductor Power Devices. New York: Wiley, 1977.

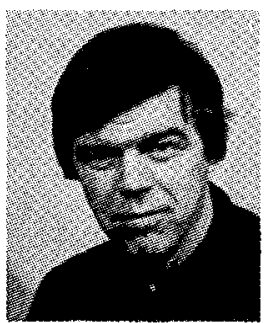

Jens Vidkjaer ( $\left.\mathrm{S}^{\prime} 72-\mathrm{M}^{\prime} 72\right)$ was born in Copenhagen, Denmark, on May 13, 1943. He received the M.S. and the Ph.D. degrees from the Technical University of Denmark in 1968 and 1975 , respectively.

Since 1970 he has been with the Electronics Laboratory, the Laboratory for Semiconductor Technology and the Electronics Institute of the Technical University of Denmark. He has been engaged in CAD of I.C.'s, numerical circuit analfier investigations 